revista.economiaypolitica@ucuenca.edu.ec Universidad de Cuenca

Ecuador

\title{
Efecto del bono de desarrollo humano en el gasto en salud y educación en Ecuador utilizando propensity score matching
}

Guamán, Jéssica; Lara, Estefanía; Alvarado, Rafael; Ponce, Pablo

Efecto del bono de desarrollo humano en el gasto en salud y educación en Ecuador utilizando propensity score matching

Revista Economía y Política, vol. XV, núm. 30, 2019

Universidad de Cuenca, Ecuador

DOl: https://doi.org/10.25097/rep.n30.2019.02

(C) Universidad de Cuenca 2019

Esta obra está bajo una Licencia Creative Commons Atribución-NoComercial-Compartirlgual 4.0 Internacional. 


\title{
Efecto del bono de desarrollo humano en el gasto en salud y educación en Ecuador utilizando propensity score matching
}

\begin{abstract}
Effect of the human development bonus on health and education expenditure in Ecuador using propensity score matching
\end{abstract}

Jéssica Guamán

DOI: https://doi.org/10.25097/rep.n30.2019.02

Universidad Nacional de Loja, Ecuador

jessicaivanova041296@gmail.com

Estefania Lara

Recepción: 15 Mayo 2019

Universidad Nacional de Loja, Ecuador

Aprobación: 22 Junio 2019

laraestefania6@gmail.com

Rafael Alvarado

Universidad Nacional de Loja, Ecuador

rafaalvaradolopez@gmail.com

(DD http://orcid.org/0000-0002-3213-5431

Pablo Ponce

Universidad Nacional de Loja, Ecuador

pablo_vpo@hotmail.com

(iD http://orcid.org/0000-0003-2717-0176.

Recepción: 15 Mayo 2019

Aprobación: 22 Junio 2019

\section{Resumen:}

El objetivo de esta investigación es evaluar el efecto del Bono de Desarrollo Humano en el gasto de salud y educación en Ecuador utilizando Propensity Score Matching. Usamos microdatos obtenidos de la Encuesta de Condiciones de Vida del 2013-2014 publicada por el INEC. Estimamos dos conjuntos de modelos, regresiones OLS y modelos de elección discreta. Los resultados muestran que los beneficiarios del BDH gastan menos en salud y educación con respecto a quienes no reciben. Los resultados muestran consistencia con la inclusión de variables de control socio demográficas. Una posible implicación de política es que la transferencia monetaria condicionada no necesariamente está cumpliendo los objetivos del programa. En consecuencia, las políticas sociales deberían garantizar que este tipo de programas no sean un instrumento de dependencia que limiten las capacidades individuales debido a que esto impide la reducción de la pobreza y la desigualdad.

Palabras Clave: Bono de Desarrollo Humano, PSM, Salud, Educación, Ecuador.

\section{ABSTRACT:}

The objective of this research is to evaluate the effect of the Human Development Bonus on health and education spending in Ecuador using Propensity Score Matching. We use microdata obtained from the Living Conditions Survey 2013-2014 published by the INEC. We estimate two sets of models, OLS regressions and discrete choice models. The results show that BDH beneficiaries spend less on health and education than those who do not. The results show consistency with the inclusion of sociodemographic control variables. One possible policy implication is that conditional cash transfer is not necessarily meeting program objectives. Consequently, social policies should ensure that these types of programs are not an instrument of dependence that limits individual capacities because this limit the reduction of poverty and inequality.

KEYWORDS: Human Development Bonus, PSM, Health, Education, Ecuador. 


\section{INTRODUCCIÓN}

Las Transferencias Condicionadas de dinero (TCD) son políticas orientadas a reducir el trabajo infantil (Del Carpio, Loayza, y Wadab, 2016), aumentar los niveles de educación y el acceso a la salud (García, Harker, y Cuartas, 2019), reducir la vulnerabilidad de los hogares (Uchiyama, 2019), entre otros. En Ecuador, desde el año 2000 se aplicó el primer programa de transferencia de dinero, cuyo fin era reducir el impacto social de la eliminación del subsidio al gas de uso doméstico. Esta política evolucionó hasta convertirse en la actualidad en una transferencia condicionada de dinero, denominada Bono de Desarrollo Humano (BDH). Las TCD requieren de una constante evaluación con el fin de asegurar que la política esté cumpliendo los objetivos, con el fin de promover la eficiencia de los recursos públicos. En este contexto, uno de los propósitos de esta investigación es evaluar esta política social en el ámbito de la salud y educación de los hijos de las madres beneficiadas de la TCD.

La entrega del BDH está condicionada a la inversión en educación y salud dirigida a los hogares más pobres. La relevancia de este programa no es solo económico y social, sino que puede tener una connotación política. En el Presupuesto General del Estado (PGE) del 2014, su participación fue de 2,08\% (715,93 millones de dólares). El número de beneficiarios representa el 9,43\% de la población total del Ecuador.

Por una parte, este programa es visto como uno de los principales logros sociales del Estado en la consecución de bienestar en la población, como lo señala la Comisión Económica para América LatinaCEPAL (2014) en el informe Panorama Social para América Latina, el cual señala que Ecuador se ubica entre los tres países más equitativos de la región, al reducir la pobreza de forma significativa durante el 2003 y el 2015. Sin embargo, existe la preocupación de que el dinero recibido por los beneficiarios del programa no necesariamente sea utilizado en la educación o atención médica de los hijos de las madres beneficiadas del $\mathrm{BDH}$. Resulta lógico pensar que el acceso a la salud y a la educación juega un rol relevante en la reducción de la pobreza y la desigualdad. Por ejemplo, López y Valdés (2000) sostienen que la pobreza se debe a la existencia de pocos activos (humanos y físicos). Estos son insuficientes en cantidad y calidad (niveles de escolaridad escasos y deficientes). Kharroubi (2007) señala que la pobreza se potencia cuando los ingresos son insuficientes para solventar los gastos en necesidades básicas. Además, es posible que las TCD pueden perpetuar la pobreza en lugar de reducirla.

Araujo, Ferreira, Lanjouw y Özler (2008) muestran que los lugares más pobres son más propensos a recibir el BDH y que la pobreza ha persistido en estos lugares. Sin un adecuado seguimiento y evaluación del programa, es posible que la población beneficiada utilice los recursos para otros fines (Fiszbein y Schady, 2009), mientras que una TCD bien enfocada puede contribuir a reducir la violencia contra las mujeres, reducir la fertilidad, la desnutrición, y mejorar la estabilidad económica de los hogares vulnerables, así como disminuir el trabajo infantil (Uchiyama, 2019; Kronebusch y Damon, 2019; Olson, Clark, y Reynolds, 2019; Del Carpio, Loayza y Wada, 2016).

La teoría del bienestar que sustenta las TCD, señala que el Estado debe proveer las condiciones necesarias para obtener el máximo bienestar social. Weber (1944) argumentó que el Estado debe establecer un conjunto de derechos sociales, tomando en cuenta las garantías individuales, la seguridad individual y familiar, y la de todos los ciudadanos sin distinción social.

La política del bienestar está basada en la aplicación de estrategias de desarrollo humano, para lograr mejoras en la calidad de vida de la población. La evidencia empírica para Ecuador sobre el BDH incluye las investigaciones de Schady y Araujo (2006), quienes enfatizan los efectos positivos y significativos en la tasa de matriculación y asistencia escolar de los hijos de los beneficiarios del programa. Asimismo, Carrillo y Jarrín (2009) mencionan que debe existir una compensación monetaria para cubrir los costos de transporte generados por la movilización de los beneficiarios de las zonas rurales para recibir el BDH. Ponce y Bedi (2010) demuestran que existe una relación significativa entre el aprendizaje y las TCD, especialmente en la población infantil. Además, Samaniego y Tejerina (2010) señalan que un objetivo dentro de la aplicación del 
BDH debe ser la capacitación financiera a las familias beneficiarias, lo cual permitiría mejorar la efectividad del programa y Fernald e Hidrobo (2011) analizan los efectos del BDH en la salud, demostrando que existe un efecto positivo en los niños de zonas rurales. Sin embargo, la mayor parte de estas investigaciones no incorporan la comparabilidad de las observaciones para asegurar que las comparaciones sean correctas.

En este contexto, la presente investigación examina los efectos del BDH en la salud y educación de los beneficiarios para Ecuador utilizando microdatos y propensity Score Matching (PSM). La hipótesis planteada es que los beneficiarios del BDH tienen un menor gasto en salud y educación, en comparación con aquellas personas que no acceden al BDH.

La presente investigación debate la efectividad del $\mathrm{BDH}$ mediante la metodología utilizada para la evaluación de los efectos del BDH en Ecuador, puesto que, no existe amplia evidencia empírica que aborde este tema con la aplicación de PSM. La ventaja del uso de PSM es que permite la comparabilidad de las observaciones y en consecuencia, de los resultados. Específicamente, permite la comparación de los resultados de educación y salud entre el grupo que recibe el BDH y el grupo que no recibe.

Los resultados de la investigación ofrecen nuevas ideas sobre la efectividad del programa y sustentan nuevas implicaciones de política. Las políticas públicas deberían estar dirigidas a efectivizar la distribución del programa, su cumplimiento, reforzar el control de los requisitos para ser beneficiarios, implementar programas de capacitación financiera para que los beneficiarios administren la transferencia de forma correcta y el dinero sea utilizado en la educación y salud (Rineharth, 2014; Patanakul, Kwak, Zwikael y Liu 2016; Churchill y Yew, 2017).

El resto de esta investigación tiene la siguiente estructura: en la segunda sección se presenta una revisión de las investigaciones previas sobre el tema, la tercera sección contiene los datos y se plantea la estrategia econométrica. En la cuarta sección se discuten los resultados encontrados, con la teoría y la evidencia empírica. La quinta sección contiene las conclusiones e implicaciones de política.

\section{REVISIÓN DE LITERATURA}

La literatura teórica que respalda las TCD es la teoría del bienestar. Una parte importante de la literatura sugiere que existe un efecto positivo de las TCD en el bienestar de las personas (Kronebusch y Damon, 2019; Olson, Clark y Reynolds, 2019). Asimismo, en el enfoque de bienestar ampliamente difundido en el siglo XX, el rol del estado es crucial para asegurar el bienestar de la población. Por ejemplo, Weber (1944) definió al Estado como el ente encargado de establecer un conjunto de derechos sociales, tomando en cuenta la seguridad individual y familiar sin distinción social, para lograr mejoras en la calidad de vida de la población. En otras palabras, la política del bienestar está basada en la aplicación de estrategias de desarrollo humano. Las TCD como parte de las políticas implementadas por el gobierno, pretenden mejorar las condiciones de vida de los grupos más vulnerables, estableciendo una relación positiva en el acceso a educación y la atención médica.

La evidencia empírica que sustenta la presente investigación se divide en tres grupos. En el primer grupo constan las investigaciones que hacen referencia a los programas de transferencias económicas que tienen como objetivo mejorar cobertura o calidad de la educación (Schady y Araujo 2006; Silva, 2015). Las investigaciones realizadas para Ecuador demuestran el impacto de las TCD en la población infantil, teniendo como resultado un efecto positivo en la matrícula escolar y en la asistencia.

Del mismo modo, las investigaciones desarrolladas por De Janvry, Finan, Sadoulet y Vakis (2006), Filmer y Schady (2011), Schimit, Monteiro y Omar (2014), Jones y Samuels (2015), Bobba y Gignoux (2016) también encuentran que las TCD tienen un efecto social esperado en los niños extremadamente pobres y vulnerables, con mayor énfasis en los niños de los deciles más pobres.

La lógica que sustenta el efecto positivo de las TCD es que los recursos obtenidos de estas, permiten a las familias financiar los costos que involucra el enviar a los hijos a la escuela y que existen políticas sociales 
complementarias que incentivan a los hijos de los beneficiarios de las transferencias a enviar a sus hijos a estudiar.

El segundo grupo de investigaciones afines con las TCD, están relacionadas con la cobertura, acceso o calidad de la salud. Schady y Paxson (2007) y Ponce y Bedi (2010) establecen efectos positivos en el desarrollo de los niños más pobres. Igualmente, Fernald, Gertler y Neufeld (2008), Rivera, Currais y Rungo (2009), Fernald e Hidrobo (2011), señalan que las TCD constituyen un instrumento adecuado para reducir la pobreza. Sin embargo, destacan que los niños de zonas rurales tienen un desarrollo de habilidades lingüísticas, pero no hay un impacto positivo y estadísticamente significativo en la salud de los beneficiarios o el efecto es limitado.

Por otro lado, Fiallos y Cantero (2008) y White y Basu (2016) afirman que los programas están dirigidos a los más pobres, pero resultan contraproducentes al provocar dependencia de los beneficiarios.

Finalmente, el tercer grupo se refiere a los programas de TCD concernientes a educación y salud en conjunto, como es el caso de los trabajos de Rineharth (2014), Patanakul, Kwak, Zwikael y Liu (2016), Churchill y Yew, (2017). Estas investigaciones mantienen cierta contrariedad con respecto a programas gubernamentales, sostienen que estos contribuyen al crecimiento nacional, sin embargo, la gestión puede resultar insuficiente y pueden ocasionar vulnerabilidad para los beneficiarios de los programas de transferencias. Por otro lado, Del Carpio, Loayza y Wada (2016) en su trabajo para Nicaragua, mencionan que el programa de TCD en zonas rurales encaminados a asistencia escolar de niños y exámenes de salud, tuvo un impacto positivo. Mientras que, Carrillo y Jarrín (2009) y, Rineharth y Mcguire (2017) consideran los costes que implica la movilización para acceder al $\mathrm{BDH}$, concluyendo que los beneficiarios de las zonas rurales deben ser compensados en una proporción relativamente mayor que en las zonas urbanas. Por último, Thompson (2014) mostró efectos positivos en la escolaridad y en la salud para quienes reciben la ayuda gubernamental.

Una parte importante de la literatura previa no considera la comparabilidad de las observaciones, generando posiblemente conclusiones sesgadas; de ahí la necesidad de asegurar que los resultados sean comparables, lo cual es posible alcanzar mediante la aplicación de métodos de emparejamiento como el PSM. La literatura sobre el BDH que incorpora métodos de emparejamiento es limitada en el contexto ecuatoriano.

\section{DAtos y Metodología}

\subsection{Fuentes estadísticas}

Con el fin de alcanzar el objetivo de la investigación, utilizamos datos individuales de corte transversal de la Encuesta de Condiciones de Vida (ECV) publicada por el Instituto Nacional de Estadísticas y Censos (INEC), Sexta Ronda (2013-2014). La Figura 1 muestra la relación entre el BDH y el gasto en educación $\mathrm{y}$ en salud respectivamente. $\mathrm{El} \mathrm{BDH}$, la variable independiente, es una variable dicótoma (es beneficiario o no del bono). El gasto en educación y salud son las variables dependientes para Ecuador. En la parte superior de la figura, podemos observar la relación de $\mathrm{BDH}$ y educación. En el primer caso, educación es una variable dicótoma y en el segundo caso educación es una variable numérica. En la parte inferior de esta figura, indicamos la relación entre el BDH y salud, donde esta variable es dicótoma. En todos los casos, el eje de las abscisas detalla si las personas son beneficiarios o no del BDH. 

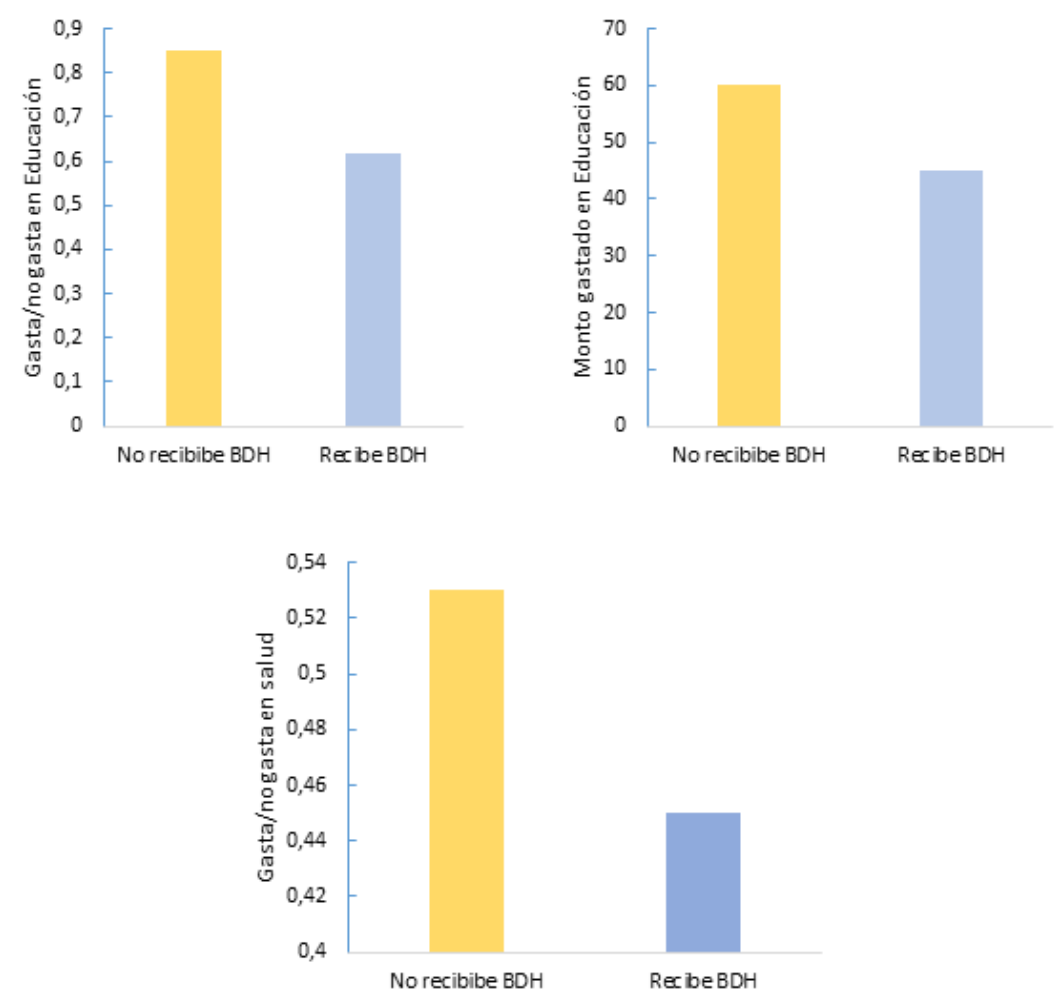

FIGURA 1.

Encuesta de Condiciones de Vida, 2013-2014

El gasto en educación representado en la figura 1 ilustra dos variables diferentes: la primera representa si realizó o no gasto en educación (panel superior izquierdo). Mientras que el panel de la derecha ilustra el monto gastado promedio en educación, los que reciben el bono y los que no reciben, respectivamente. Las personas que no reciben el BDH tienen un gasto en educación mayor que las personas que son beneficiarias del programa. Asimismo, el panel inferior ilustra si gasta o no gasta en salud los beneficiarios del programa y quienes no lo reciben. Sin embargo, en la práctica, las personas que reciben el BDH con las que no reciben no son comparables, por lo que es necesario el método de emparejamiento para asegurar la comparabilidad de las observaciones. Esto se explica porque las personas que reciben el bono están bajo la línea de la pobreza y con el propósito de reinserción escolar y asistencia continua a clases, el gobierno ha proporcionado uniformes escolares, textos a las instituciones educativas y desayunos escolares, contribuyendo de esta manera, a disminuir el gasto de las familias.

La Tabla 1 muestra el cuadro resumen de las variables estimadas en el modelo. La variable independiente BDH y las variables de control: edad, sexo, área, idioma, etnia, región natural y estado civil, cuentan con 65370 observaciones. Mientras, que el gasto en salud y educación (numérica y dicótoma) cuentan con 19235, 9008 y 10508 observaciones respectivamente. En los estadísticos descriptivos, la media (Mean) representa en términos generales el promedio del conjunto de observaciones. La desviación estándar (DS) que no supera a uno en las variables de $\mathrm{BDH}$, gasto en salud, gasto en educación (dicótoma), sexo, área, idioma, región natural y estado civil. Es decir, no se encuentran muy alejadas de la media. Las variables de gasto en educación (numérica), edad y etnia tienen una desviación estándar elevada. 
TABLA 1.

Estadísticos descriptivos de las variables

\begin{tabular}{llllll}
\hline Máx. & Mín. & DS & Media & N & Variables \\
\hline 11 & 00 & 0,39 & 0,18 & 65370 & BDH Gasto en \\
1001 & 10 & 0,49 & 0,51 & 19235 & salud Gasto \\
& & 62,79 & 59,75 & 9008 & en educación \\
& & 0,34 & 0,85 & 10508 & Gasto en \\
& & & & & educación* \\
651 & 150 & 14,01 & 35,02 & 65370 & Edad Sexo \\
11 & 00 & 0,49 & 0,48 & 65370 & Area Idioma \\
& & 0,49 & 0,48 & 65370 & \\
441 & 11 & 0,26 & 0,07 & 65370 & \\
& 0 & 0,79 & 3,47 & 65370 & Etnia Región \\
& & 0,48 & 0,37 & 65370 & natural \\
\hline
\end{tabular}

Encuesta de Condiciones de Vida, 2013-2014

\subsection{Metodología}

En la práctica, la técnica PSM construye un grupo de comparación estadística que se basa en un modelo de la probabilidad de participar en el tratamiento, utilizando las características observadas. Los participantes son emparejados sobre la base de esta probabilidad (puntaje de propensión) a los no partícipes del programa. El efecto medio del tratamiento del programa se calcula como la diferencia media en los resultados entre estos dos grupos. La validez del PSM depende de la independencia condicional y un apoyo común considerable en las puntuaciones de propensión a través de las muestras participantes y no participantes. Autores como Ponce, Robles, Alvarado \& Ortiz (2019), utilizaron el PSM para determinar el efecto del capital humano en la brecha de ingresos en Ecuador entre grupos étnicos y por el sexo. El PSM es una técnica útil cuando se cree que las características observadas afectan la participación del programa. El pionero de esta técnica fue Rubin (1974), sobre estas investigaciones se desarrollaría más tarde la técnica PSM como tal. Posteriormente, el puntaje de propensión es definido por Rosenbaum y Rubin (1983) como la probabilidad condicional de recibir un tratamiento dadas características de pretratamiento:

$$
P(X) \equiv \operatorname{Pr}(D=1 \mid X)=E(D \mid X)
$$

Rosenbaum y Rubin (1983) demostraron que se obtienen los mismos resultados, si en vez de condicionar las distribuciones de la variable de resultados por el vector de características $\mathrm{X}$, se controla únicamente por la probabilidad de ser tratado $\mathrm{p}(\mathrm{X})$, lo que acaba con el problema de dimensionalidad. Como resultado, dada una población de unidades denotada por i, si se conoce la puntuación de propensión $\mathrm{p}(\mathrm{Xi})$, entonces el Efecto Promedio del Tratamiento en el Tratado (ATT) puede estimarse como sigue:

$$
\begin{gathered}
\tau=E\left\{Y_{1 i}-Y_{0 i} \mid D_{i}=1\right\} \\
\tau=E\left[E\left\{Y_{1 i}-Y_{0 i}\left|D_{i}-Y_{0 i}\right| D_{i}=1, p\left(X_{i}\right)\right\}\right]
\end{gathered}
$$




$$
\begin{gathered}
\tau=E\left[E\left\{Y_{1 i} \mid D_{i}=1, p\left(X_{i}\right)\right\}-E\left\{Y_{0 i} \mid D_{i}=0, p\left(X_{i}\right)\right\} \mid D_{i}=1\right] \\
E\left\{Y, I T_{i}=j, p\left(X_{i}\right)\right\}, \text { para } \mathrm{j}=0,1
\end{gathered}
$$

Chen, Mu y Ravallion (2008) describen una aplicación de este enfoque. Permite que las ventajas de consistencia de la concordancia se combinen con las propiedades de varianza favorables de los métodos basados en regresión. Las ecuaciones (5) y (6) estiman el efecto del tratamiento sobre las personas participantes en el programa estatal BDH. En estas ecuaciones se estiman regresiones paramétrica y no paramétrica respectivamente. En la estimación no paramétrica de la ecuación (5) la variable dependiente es el monto gastado en educación y para la estimación paramétrica en la misma ecuación, la variable dependiente representa si han gastado o no en materiales escolares. Por otro lado, en la estimación paramétrica de la ecuación (6) la variable dependiente indica si las personas han realizado gasto en salud o no. En lo que se refiere a la variable independiente representa al número de personas que reciben o no el $\mathrm{BDH}$ en las ecuaciones (5) y (6). Se requiere un modelo logit para la estimación paramétrica y una regresión lineal para la no paramétrica. Estimamos un modelo independiente para cada variable, como se indica a continuación:

$$
\begin{gathered}
\text { Educación }_{i}=\beta_{0}+\beta_{1} B D H_{i}+\varepsilon_{i} \\
\text { Salud }_{i}=\beta_{0}+\beta_{1} B D H_{i}+\varepsilon_{i}
\end{gathered}
$$

En las ecuaciones (5) y (6)BDH $H_{i}$ es una variable dicótoma que representa el número de personas que reciben o no el $\mathrm{BDH}$, y $\varepsilon$ i es el término de error estocástico. En la ecuación (6)Educación ${ }_{i}$ está determinado por el monto gastado en textos y materiales escolares, en la estimación no paramétrica. $Y$ en la estimación paramétrica Educación ${ }_{i}$ representa si gasta/no gasta en educación medido por el gasto en textos y materiales escolares. Finalmente, en la ecuación (7) Salud ${ }_{i}$ es una variable dicótoma y está determinada por el gasto/no gasto en salud. Para tener un contexto más amplio de la investigación incluimos variables de control, mismas que se presentan en las siguientes ecuaciones de acuerdo con cada caso.

$$
\begin{aligned}
& \text { Educación }_{i}=\beta_{0}+\beta_{1} B^{B D H_{i}}+\beta_{2} Z_{i}+\varepsilon_{i} \\
& \text { Salud }_{i}=\beta_{0}+\beta_{1} B D H_{i}+\beta_{2} Z_{i}+\varepsilon_{i}
\end{aligned}
$$

Las ecuaciones (8) y (9) son una extensión de las ecuaciones (6) y (7) respectivamente, en las que se agrega variables de control determinadas por $Z_{i}$ que es el conjunto de covariantes adicionales que recogen el efecto 
de las características estructurales del BDH en Ecuador. Estas variables de control son: edad, sexo, área, etnia, idioma, estado civil, etnia y región natural.

\section{Discusión de Resultados}

La Tabla 2 muestra los resultados del BDH para gastos en educación y salud representadas por las ecuaciones (6) y (7) en Ecuador. Los resultados indican una relación negativa y estadísticamente significativa entre BDH y gastos en educación y salud para los beneficiarios en la estimación no paramétrica y en las paramétricas. El $\mathrm{BDH}$ tiene un mayor efecto en el gasto de educación que para el de salud, esto coincide con la Figura 1. La primera columna, muestra los resultados de la estimación no paramétrica con respecto al gasto en educación, donde la elasticidad del BDH es de -16,92. Es decir, cuando una persona recibe el BDH gasta menos que las personas que no lo reciben. En las siguientes columnas se presentan los resultados de las estimaciones paramétricas del gasto en educación y salud respectivamente. La segunda columna, indica que la probabilidad de gastar en materiales escolares es $0,22 \%$ veces menos, cuando una persona recibe el BDH. Finalmente, la tercera columna, muestra que la probabilidad de gastar en atención médica es $0,09 \%$ veces menos cuando una persona es beneficiaria del BDH. Estos resultados son estadísticamente significativos.

TABLA 2.

Resultados del BDH con relación a educación y salud en Ecuador

\begin{tabular}{llll}
\hline & \multicolumn{2}{c}{ No } & \\
& Paramétrico & Paramétrico \\
\hline & Educación & Educación & Salud \\
\hline BDH & $-16,92$ & $-0,22$ & $-0,09$ \\
& $(-3,83)$ & $(-8,38)$ & $(-11,92)$ \\
Constante & 60,15 & & \\
& $(89,92)$ & & \\
\hline Observaciones & 9008 & 10508 & 19235 \\
R2 Ajustado & 0.002 & & \\
\hline
\end{tabular}

Encuesta de Condiciones de Vida, 2014

Si los valores t son mayores a 2 en valores absolutos, los coeficientes son estadísticamente significativos.

Los resultados señalan que el gasto en educación tiene mayor elasticidad que el gasto en salud, en las estimaciones paramétrica y no paramétrica. Esto estaría explicado ya que la mayoría de los beneficiarios destinan el dinero principalmente a cubrir los gastos en la educación de sus hijos, durante el periodo escolar. Mientras que, los gastos en salud no representan un rubro permanente para las familias como el caso de la educación. Sin embargo, algunas personas pueden utilizar el BDH para otros gastos como: compra de alimentos, vestimenta, pago de deudas, inversión en negocio propio, ahorro, etc. Es decir, el BDH no se destina a cubrir únicamente los gastos condicionados.

El ajuste del modelo, medido por $R^{2}$ es bajo para educación no paramétrica. Es decir, la capacidad explicativa de los cambios en la variable dependiente está levemente explicada por las variaciones en la variable independiente. Las estimaciones paramétricas, no presentan valores $R^{2}$ debido a que utilizamos modelos logit. Los resultados de las estimaciones están respaldados por la teoría del bienestar, Weber (1944) señala que una de las funciones del Estado es establecer derechos sociales para promover adecuadas condiciones de vida para la población. El BDH como un programa enfocado en la protección de los más vulnerables, ha tenido un efecto significativo en los beneficiarios. La disyuntiva se origina en el destino que se le da al BDH, debido a que, el consumo de las familias aumenta y mejoran sus condiciones de vida. Sin embargo, puede generar dependencia y ser un desincentivo para la superación del individuo como tal, ocasionando pérdidas económicas para el Estado. 
Los resultados obtenidos de la Tabla 2 son opuestos a los resultados obtenidos en la investigación de Fiallos y Cantero (2008), donde hacen referencia a que los programas en salud dirigidos a los más pobres mejoran su estado emocional y apoya los programas asistenciales exitosos como la complementación alimentaria. Sin embargo, resultan contraproducentes si no cumplen con la premisa de eliminar inequidades sociales. Entonces, las ayudas estatales tendrían un efecto negativo y podrían generar dependencia. Por otro lado, los resultados difieren de los resultados encontrados en los trabajos de Schady y Araujo (2006), Schady y Paxson (2007), Ponce y Bedi (2010), Silva (2015). En estas investigaciones desarrolladas para Ecuador, las TCD tienen un efecto positivo para los beneficiarios y demuestran que, el programa $\mathrm{BDH}$ tuvo gran impacto especialmente en el desarrollo infantil.

De igual manera, las investigaciones de Rivera, Currais y Rungo (2009), Firpo, Pieri, Pedroso, Souza (2014) y Schimit, Monteiro y Omar (2014), fueron desarrolladas en torno al Programa Bolsa Familia en Brasil. En el primero, los autores señalan un gran impacto en educación y reducción de la pobreza, esto permite predecir mejoras en el estado de salud de la población a largo plazo. En el segundo, encontraron pruebas de que los individuos manipulan sus ingresos reduciendo voluntariamente su oferta de mano de obra para poder ser elegibles en el programa. Y en la tercera investigación, se recalca la importancia de la inversión del gobierno en la clase baja desempleada para un crecimiento de la clase media.

En la investigación de Filmer y Schady (2011) para Camboya encontraron que una modesta transferencia de efectivo, equivalente a aproximadamente el $2 \%$ del consumo del hogar mediano receptor, tuvo un impacto sustancial en la asistencia escolar. En el programa Zomba Cash Transfer en el sur de Malawi, Thompson (2014) mostró aumentos en la asistencia escolar, la salud mental y apoyo psicosocial, para las personas más vulnerables. Sin embargo, la diferencia no es significativa entre los receptores condicionados y no condicionados de transferencias en efectivo. Esto se relaciona con Ecuador, porque algunos beneficiarios utilizan el bono para gastos distintos a los condicionados, entonces las TDC pueden promover indirectamente el bienestar, aunque el dinero no se destine a los gastos previstos.

En investigaciones para México De Janvry, Finan, Sadoulet y Vakis (2006), Fernald, Gertler y Neufeld (2008) y, Bobba y Gignoux (2016) mencionan que, las transferencias al hogar están asociadas con resultados significativamente mejores en muchos aspectos del niño. En esta tendencia, Fernald e Hidrobo (2011) y Jones y Samuels (2015) coinciden en que los efectos de las transferencias de efectivo en la escolaridad y salud de los niños extremadamente pobres y vulnerables, que generalmente se encuentran en zonas rurales, son positivos.

Finalmente, en el trabajo para Nicaragua, Del Carpio, Loayza y Wada (2016) manifiestan que el programa social tuvo un impacto positivo, aumentó la asistencia escolar y los chequeos médicos. Por su parte, White y Basu (2016) señalan que en Perú, una disminución de la frecuencia de los pagos está relacionado con un desbalance nutritivo en los beneficiarios. Además, Patanakul, Hoon, Zwikael y Liu (2016) sostienen que los proyectos y programas gubernamentales contribuyen al crecimiento nacional en gran magnitud. Sin embargo, los registros muestran que no están bien administrados o controlados sobre el fin que tiene dicha ayuda gubernamental. Ecuador, también debería implementar mejoras al sistema de control y elección de los beneficiarios. Por tanto, Churchill y Yew (2017) consideran que el impacto de las transferencias gubernamentales depende del desarrollo de los países. Las TCD son perjudiciales para el crecimiento económico en los países desarrollados. Por tanto, en Ecuador, al ser un país en vías de desarrollo, el impacto de dichas transferencias es positivo y aumenta el crecimiento.

A continuación, se muestra una distribución de Kernel tanto para el gasto en educación como para el gasto en salud con respecto al BDH. La distribución forma parte de la metodología PSM tanto no paramétrica como paramétrica de nuestra investigación. 
A

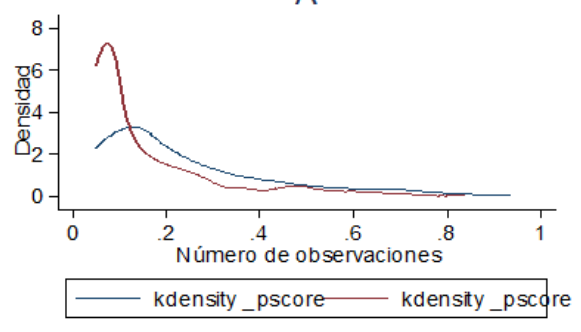

C

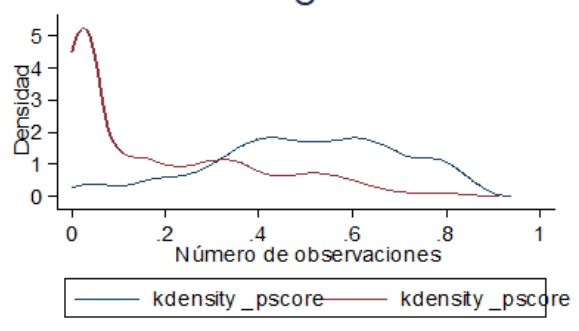

B

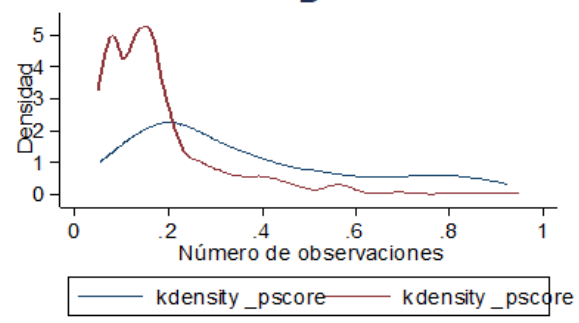

FIGURA 2.

Distribución de Kernel

Encuesta de condiciones de vida, 2014

La Figura 2 muestra la distribución de Kernel del BDH para el gasto en educación y en salud respectivamente. El área bajo la intersección de los grupos se denomina Base de Soporte Común (BSC). En los paneles (A) y (B) observamos el comportamiento del BDH con respecto a la educación aplicando PSM no paramétrico y paramétrico respectivamente. En donde el área de la BSC es considerable. Es decir, son representativas. Por otro lado, en el panel (C) observamos el BDH con respecto a la salud utilizando PSM paramétrico, donde la BSC también es considerable. De igual manera, este modelo es significativamente representativo.

La Tabla 3 muestra los resultados de la estimación del BDH tanto para el gasto en educación como para el gasto en salud incluyendo variables de control, utilizando PSM. En las primeras columnas, se presentan los resultados del gasto en educación con respecto al $\mathrm{BDH}$, con estimaciones no paramétricas.

En la primera, se muestran los resultados Sin Variables Comparables (SVC), en donde, las personas que reciben el $\mathrm{BDH}$ gastan $\$ 18,98$ menos en educación que los no beneficiarios. En la segunda, los resultados aumentan considerablemente a \$45,47 después de la aplicación del PSM, es decir, Con Variables Comparables (CVC); por tanto, se ratifica el supuesto de que los beneficiarios del bono realizan un gasto menor en educación. Y en la tercera, se presentan las variaciones encontradas en los resultados SVC y CVC. En esta columna se demuestra que los resultados iniciales estaban sesgados en 58,23\% del gasto en educación de las personas que reciben el BDH y las que no son beneficiarias.

En las columnas siguientes se muestran las estimaciones paramétricas para el gasto en educación y en salud con respecto al BDH. Las variables dependientes utilizadas son dicótomas, por tanto, aplicamos modelos LOGIT. En las tres primeras, presentamos los resultados de las estimaciones con gasto en educación. En la primera, encontramos resultados SVC, donde las personas que reciben el BDH tienen una probabilidad menor de $0,01 \%$ de gastar en materiales escolares en comparación a quienes no reciben el bono. En la segunda, CVC los resultados aumentan a $0,02 \%$. Es decir, que aplicando PSM la probabilidad de gastar en materiales escolares sigue siendo mayor para quienes no acceden al bono. Y en la tercera, la variación entre SVC y CVC muestran que, al inicio los resultados estaban sesgados en $57,69 \%$ en la probabilidad de gasto en educación para quienes reciben y las que no reciben el $\mathrm{BDH}$.

Las tres últimas columnas presentan resultados del gasto en salud (realizó gasto en salud o no). En la primera, los resultados SVC indican que las personas que reciben el BDH tienen una probabilidad de 0,08\% 
menor de efectuar gastos en atención médica con respecto a que los que no reciben el bono. En la segunda columna, CVC el coeficiente del BDH registra una probabilidad del 0,09\% menor a realizar gastos en salud. En la última columna, con la variación entre SVC y CVC, se entiende que inicialmente existía un sesgo de $12,94 \%$ en las estimaciones de gasto en salud de los beneficiarios del bono y los que no lo son.

El sesgo es superior al 50\% afectando considerablemente a las variables de control. En los modelos paramétricos, los resultados obtenidos con el gasto en educación al utilizar PSM con variables de control, son estadísticamente significativas: edad y área. En el caso de salud, al aplicar PSM las variables significativas son: edad, área, idioma, estado civil y región natural. La inclusión de las variables de control no afectó la significancia de la variable $\mathrm{BDH}$ con respecto al gasto en educación y salud. Se mantiene la relación negativa con un impacto positivo para las beneficiarias del BDH. Por lo tanto, las estimaciones son consistentes con el marco teórico y empírico anteriormente señalado.

TABLA 3.

Contraste entre regresiones sin variables comparables y PSM para Ecuador

\begin{tabular}{|c|c|c|c|c|c|c|c|c|c|}
\hline \multicolumn{6}{|c|}{ PARAMÉTRICO (LOGIT) } & \multicolumn{4}{|c|}{ NO PARAMÉTRICO } \\
\hline $\begin{array}{l}\text { Variación } \\
\text { (\%) }\end{array}$ & $\begin{array}{l}\text { Salud } \\
\text { (CVC) }\end{array}$ & $\begin{array}{l}\text { Salud } \\
\text { (SVC) }\end{array}$ & $\begin{array}{l}\text { Variación } \\
\text { (\%) }\end{array}$ & $\begin{array}{l}\text { Educación } \\
\text { (CVC) }\end{array}$ & $\begin{array}{l}\text { Educación } \\
\text { (SVC) }\end{array}$ & $\begin{array}{l}\text { Variación } \\
\text { (\%) }\end{array}$ & $\begin{array}{l}\text { Educación } \\
\text { (CVC) }\end{array}$ & $\begin{array}{l}\text { Educación } \\
\text { (SVC) }\end{array}$ & \\
\hline 12,94 & $\begin{array}{l}-0,10 \\
(-6,13)\end{array}$ & $\begin{array}{l}-0,08 \\
(-8,26)\end{array}$ & 57,69 & $\begin{array}{l}-0,03 \\
(-0,38)\end{array}$ & $\begin{array}{l}-0,01 \\
(-0,25)\end{array}$ & 58,23 & $\begin{array}{l}-45,47 \\
(-2,37)\end{array}$ & $\begin{array}{l}-18,98 \\
(-4,11)\end{array}$ & $\mathrm{BDH}$ \\
\hline 11,11 & $\begin{array}{l}0,002 \\
(3.30)\end{array}$ & $\begin{array}{l}0,00 \\
(5,65)\end{array}$ & 24,00 & $\begin{array}{l}-0,03 \\
(-14,57)\end{array}$ & $\begin{array}{l}-0,02 \\
(-17,03)\end{array}$ & 50,17 & $\begin{array}{l}0,88 \\
(1,32)\end{array}$ & $\begin{array}{l}0,44 \\
(3,01)\end{array}$ & Edad \\
\hline 33,33 & $\begin{array}{l}-0,09 \\
(-1,09)\end{array}$ & $\begin{array}{l}0,02 \\
(0,33)\end{array}$ & 45,06 & $\begin{array}{l}0,09 \\
(1,19)\end{array}$ & $\begin{array}{l}0,05 \\
(1,07)\end{array}$ & 81,28 & $\begin{array}{l}-20,77 \\
(-1,24)\end{array}$ & $\begin{array}{l}-3,88 \\
(-2,96)\end{array}$ & Sexo (hombre) \\
\hline 3,84 & $\begin{array}{l}0,07 \\
(5,8)\end{array}$ & $\begin{array}{l}0,08 \\
(9,78)\end{array}$ & 33,33 & $\begin{array}{l}0,13 \\
(2,39)\end{array}$ & $\begin{array}{l}0,09 \\
(2,55)\end{array}$ & 38,53 & $\begin{array}{l}11,92 \\
(1,46)\end{array}$ & $\begin{array}{l}-19,39 \\
(-13,45)\end{array}$ & Área (urbano) \\
\hline 3,65 & $\begin{array}{l}-0,08 \\
(-2,56)\end{array}$ & $\begin{array}{l}-0,08 \\
(-3,77)\end{array}$ & 5,77 & $\begin{array}{l}-0,05 \\
(-0,80)\end{array}$ & $\begin{array}{l}-0,05 \\
(-1,11)\end{array}$ & 71,33 & $\begin{array}{l}12,32 \\
(1,02)\end{array}$ & $\begin{array}{l}-3,53 \\
(1,07)\end{array}$ & $\begin{array}{l}\text { Idioma } \\
\text { (indígena) }\end{array}$ \\
\hline 35,48 & $\begin{array}{l}0,03 \\
(2,01)\end{array}$ & $\begin{array}{l}0,02 \\
(2,39)\end{array}$ & 21,51 & $\begin{array}{l}-0,09 \\
(-1,28)\end{array}$ & $\begin{array}{l}-0,07 \\
(-1,72)\end{array}$ & 74,39 & $\begin{array}{l}9,84 \\
(0,60)\end{array}$ & $\begin{array}{l}-2,52 \\
(-0,71)\end{array}$ & $\begin{array}{l}\text { Estado civil } \\
\text { (soltero) }\end{array}$ \\
\hline 42,60 & $\begin{array}{l}0,07 \\
(1,26)\end{array}$ & $\begin{array}{l}0,12 \\
(4,29)\end{array}$ & 73,33 & $\begin{array}{l}0,05 \\
(0,31)\end{array}$ & $\begin{array}{l}0,01 \\
(0,12)\end{array}$ & 60,17 & $\begin{array}{l}-24,91 \\
(-0,90)\end{array}$ & $\begin{array}{l}9,92 \\
(1,82)\end{array}$ & $\begin{array}{l}\text { 2.Etnia } \\
\text { (blanco) }\end{array}$ \\
\hline 41,33 & $\begin{array}{l}0,04 \\
(1,11)\end{array}$ & $\begin{array}{l}0,08 \\
(3,36)\end{array}$ & 53,59 & $\begin{array}{l}0,18 \\
(0,86)\end{array}$ & $\begin{array}{l}0,08 \\
(0,74)\end{array}$ & 65,68 & $\begin{array}{l}-20,04 \\
(-1,01)\end{array}$ & $\begin{array}{l}6,87 \\
(1,47)\end{array}$ & $\begin{array}{l}\text { 3.Etnia } \\
\text { (montubio) }\end{array}$ \\
\hline 16,94 & 0,05 & 0,06 & 22,22 & $-0,04$ & $-0,05$ & 0,632 & 6,48 & 6,44 & $\begin{array}{l}\text { 4.Etnia(afro, } \\
\text { negro, } \\
\text { mestizo) }\end{array}$ \\
\hline 23,52 & $-0,02$ & $-0,01$ & 63,21 & 0,10 & 0,04 & 95,36 & $-0,42$ & $-9,05$ & $\begin{array}{l}\text { 2.Región } \\
\text { natural } \\
\text { (Costa) }\end{array}$ \\
\hline & $(-0,97)$ & $(-1,53)$ & & $(1,81)$ & $(1,12)$ & & $(-0,02)$ & $(-5,67)$ & \\
\hline 17,08 & $-0,20$ & $-0,17$ & 95,74 & 0,05 & $-0,00$ & 22,69 & 2,45 & $-1,89$ & $\begin{array}{l}\text { 3. Reg. Nat. } \\
\text { (Amazonia) }\end{array}$ \\
\hline & $(-10,69)$ & $(-15,27)$ & 73,66 & $(0,83)$ & $\begin{array}{l}(-0,08) \\
1,27 \\
(33,56)\end{array}$ & 17,85 & $\begin{array}{l}(0,23) \\
34,1 \\
(1,84)\end{array}$ & $\begin{array}{l}(-1,01) \\
41,51 \\
(12,09)\end{array}$ & Constante \\
\hline & 19235 & 19235 & & 10327 & 10327 & & $\begin{array}{l}8874 \\
0,063\end{array}$ & $\begin{array}{l}9008 \\
0,035\end{array}$ & $\begin{array}{l}\text { Observaciones } \\
\text { R2 Ajustado }\end{array}$ \\
\hline
\end{tabular}

Encuesta de Condiciones de Vida, 2013-2014

Si los valores t son mayores a 2 en valores absolutos, los coeficientes con estadísticamente significativos.

Los resultados obtenidos, después de la inclusión de variables de control y de diferenciar variables comparables y no comparables, concuerdan con las investigaciones señaladas en los resultados de la Tabla 2 en las que existe un efecto positivo en la aplicación de programas de TDC por parte del Estado. Mientras 
que, la presente investigación difiere en el caso de Fiallos y Cantero (2008) en la que manifiesta que estos programas resultan contraproducentes y podrían generar dependencias a los beneficiarios.

Se enfatiza que los programas de transferencias gubernamentales deben ser mejor administrados para lograr una mayor eficacia. Carrillo y Jarrín (2009) sugieren mejoras al BDH, señalan que los pagos podrían ser geográficamente diferenciados de modo que el valor total del programa sea el mismo para todos los hogares. Esto sugiere que puede haber complementariedades en la movilización desde el sector rural al centro urbano. Se debería incluir a los beneficiarios en programas de educación financiera con respecto al ahorro, de acuerdo a Samaniego y Tejerina (2010). Algunos autores opinan que las transferencias no condicionadas mejorarán el bienestar de las familias pobres, independientemente de cómo se gaste el dinero.

Entre las investigaciones más recientes referentes a las TCD que coinciden con los resultados obtenidos en la presente investigación se encuentran: Gutierrez, Juarez y Rubli (2016), Alcaraz, Chiquiar, Orraca y Salcedo (2016), Alderman, Behrman y Tasneem (2017), estas fueron realizadas para México.

La aplicación de programas estatales tiene efectos positivos y estadísticamente significativos. La primera, evidencia que las transferencias públicas para ancianos son compartidas con los más jóvenes mediante inversiones en capital humano. La segunda se centra en los efectos de salud pública en las tasas de matriculación y rendimiento escolar. El aumento de matrículas escolares y una disminución de trabajo infantil demuestran que los programas estatales constituyen un componente fundamental para el desarrollo. Y en la tercera, los autores sostienen que los objetivos de estos programas no son solamente aumentar las inversiones de capital humano con implicaciones para niveles futuros, sino también reducir la pobreza y la desigualdad de ingresos

Evans, Holtemeyer y Kosec (2017), Beasley y Huillery (2016) en sus trabajos correspondientes a Tanzania y Níger respectivamente, resaltan los efectos de las TCD en salud y educación. En Tanzania, el efecto fue significativo en la aceptación del seguro de salud y una mejora en la atención médica. En Níger, las subvenciones escolares muestran que los padres aumentaron la participación y responsabilidad, pero estos esfuerzos no mejoraron la calidad de la educación. Mientras que, las investigaciones de Brune, Giné, Goldberg y Yang (2017) y Kilburn, Handa, Angeles, Mvula y Tsoka (2017) aplicadas en Malawi acera de transferencias en efectivo se manifiestan los siguientes resultados. Los primeros autores, mencionan que los esquemas de transferencia suelen estar diseñados para mejorar el bienestar, pero demostraron que ni los incumplimientos de los ahorros ni los retrasos en los pagos por parte del Estado afectan la cantidad o la composición del gasto. Por otro lado, los demás autores analizan el impacto de un ingreso positivo en los resultados de la educación infantil del Programa de Transferencia Social en Efectivo. Y manifestaron que este programa mejora las tasas de matrícula y disminuye las deserciones. Sin embargo, la principal vía intermedia entre el programa y la escolaridad es el gasto en educación. En general, los programas estatales son fundamentales para el desarrollo de la población, especialmente para la más joven.

En lo que respecta a las implicaciones de política relacionadas con las TCD, la evidencia empírica para Nicaragua de Dammert (2009) sostiene que los programas sociales tienen efectos diferenciales para cualquier subpoblación definida por covariables. Es importante conocer si los impactos de un programa están concentrados entre unas pocas personas, para alcanzar su población objetivo. Attanasio y Lechene (2014) en su investigación, los programas de TCD ofrecen una explicación para el gran cambio en el consumo total que sigue a la inyección de efectivo implícita en estos programas. Y para Piperata, McSweeney y Murrieta (2016) al analizar el Programa Bolsa Familia de Brasil sostienen que es notable que se haya convertido en un modelo global de mitigación de la pobreza a pesar del escaso escrutinio de su desempeño con respecto a sus propios objetivos programáticos y con serias limitaciones geográficas en su evaluación. Por lo tanto, reiteramos el control de este tipo de ayuda estatal.

En general, algunas de las variables de control resultan no estadísticamente significativas. En el caso del modelo no paramétrico del gasto en educación, ninguna variable de control es estadísticamente significativa luego de aplicar PSM. En las estimaciones paramétricas del gasto en educación, no son estadísticamente 
significativas: sexo, idioma, estado civil, etnia y región natural. En lo que respecta al gasto en salud, no son estadísticamente significativas: sexo, etnia y todas las regiones naturales con variables comparables. Esto no significa que estas no tengan validez. En la práctica se debe considerar la importancia de estas variables en el contexto de aplicación. Para terminar, el ajuste del modelo medido por $R^{2}$ es bajo en Ecuador después de incorporar variables de control y de realizar PSM. Este ajuste da a conocer que la capacidad explicativa de los cambios en la variable dependiente está ligeramente explicada por las variaciones en las variables independientes con respecto al gasto en educación. En el caso del gasto en educación y salud paramétrico se aplica logit, por tanto, no contamos con este valor.

\section{Conclusiones}

En la presente investigación se examinó el efecto del $\mathrm{BDH}$ con el gasto en educación y en salud, utilizando la técnica PSM paramétrico y no paramétrico. Evaluamos esta política social implementada por el gobierno, dirigida a proporcionar una TCD. Los resultados obtenidos, muestran la relación negativa y significativa del $\mathrm{BDH}$ en el gasto en educación y salud de las personas que son beneficiarias de este programa. Además, de que el $\mathrm{BDH}$ tiene una relación negativa, tiene una mayor elasticidad con respecto a la variable gasto en educación. Aplicando el método PSM la variación CVC es mayor en el caso de la educación, representando que los beneficiarios gastan menos. En la variable, gasto en salud, la variación no es tan marcada. Los resultados obtenidos, concuerdan con la mayor parte de la evidencia empírica expuesta anteriormente, la misma que señala el efecto positivo de las TCD. Se reafirma la evidencia teórica del papel del Estado, de asegurar el bienestar especialmente de los grupos más vulnerables.

Las políticas deben ir encaminadas a reforzar y asegurar una adecuada distribución de esta ayuda económica; verificar el correcto cumplimiento de los requisitos básicos de elegibilidad. Coincidiendo con Patanakul, Hoon, Zwikael y Liu (2016) se debería implementar programas paralelos de capacitación para la administración financiera en los hogares de los beneficiarios. Una política que debería implementarse es la constante actualización de los datos de los beneficiarios y el seguimiento del BDH para garantizar su funcionamiento a largo plazo. Un punto a considerar es que, los beneficiarios gastan menos porque los servicios de educación y salud, en los últimos años, han ampliado la cobertura y existe un mayor acceso a estos de forma gratuita. Por tanto, las familias están destinando el monto del $\mathrm{BDH}$ para otro tipo de rubros.

Es importante, que el $\mathrm{BDH}$ se suministre de tal manera que este no se convierta en un instrumento de dependencia económica, como lo considera Fiallos y Cantero (2008), que limite la capacidad de superación y emprendimiento de las familias y puedan salir de la pobreza.

En el desarrollo de la presente investigación se presentaron algunas limitaciones, entre ellas, la falta de variables que indiquen el monto de gasto en salud para realizar una comparación como en el caso del gasto en educación. En lo que respecta a investigaciones futuras sugerimos evaluar los posibles efectos de una disminución o aumento del monto en el BDH, para el análisis de las consecuencias de estos cambios. Una extensión de nuestra investigación puede centrarse en medir las consecuencias derivadas de la aplicación de transferencias no condicionadas de dinero. Finalmente, un estudio que evalúe las TCD y la dependencia económica en las familias receptoras.

\section{REFERENCIAS BIBLIOGRÁFICAS}

Alcaraz, C., Chiquiar, D., Orraca, M. J., \& Salcedo, A. (2016). The effect of publicly provided health insurance on education outcomes in Mexico. The World Bank Economic Review, 30 (Supplement_1), S145-S156.

Alderman, H., Behrman, J. R. (2017). The contribution of increased equity to the estimated social benefits from a transfer program: an illustration from PROGRESA/oportunidades. The World Bank Economic Review, LHX 006. 
Araujo, M. C., Ferreira, F. H., Lanjouw, P., \& Özler, B. (2008). Local inequality and project choice: Theory and evidence from Ecuador. Journal of Public Economics, 92(5), 1022-1046.

Attanasio, O. P., \& Lechene, V. (2014). Efficient responses to targeted cash transfers. Journal of Political Economy, 122(1), 178-222.

Beasley, E., \& Huillery, E. (2016). Willing but Unable? Short-Term Experimental Evidence on Parent Empowerment and School Quality. The World Bank Economic Review, lhv064

Bobba, M., \& Gignoux, J. (2016). Neighborhood effects in integrated social policies. The World Bank Economic Review, lhw061.

Brune, L., Giné, X., Goldberg, J., \& Yang, D. (2017). Savings defaults and payment delays for cash transfers: Field experimental evidence from Malawi. Journal of Development Economics.

Carrillo, P. E., \& Jarrín, J. P. (2009). Efficient delivery of subsidies to the poor: Improving the design of a cash transfer program in Ecuador. Journal of Development Economics, 90(2), 276-284.

CEPAL, Panorama Social de América Latina. (2014). Santiago de Chile; 2014.

Churchill, S. A., \& Yew, S. L. (2017). Are government transfers harmful to economic growth? A meta-analysis. Economic Modelling, 64, 270-287.

Dammert, A. C. (2009). Heterogeneous impacts of conditional cash transfers: Evidence from Nicaragua. Economic Development and Cultural Change, 58(1), 53-83.

De Janvry, A., Finan, F., Sadoulet, E., \& Vakis, R. (2006). Can conditional cash transfer programs serve as safety nets in keeping children at school and from working when exposed to shocks? Journal of Development Economics, 79(2), 349-373.

Del Carpio, X. V., Loayza, N. V., \& Wada, T. (2016). The Impact of Conditional Cash Transfers on the Amount and Type of Child Labor. World Development, 80, 33-47

Evans, D. K., Holtemeyer, B., \& Kosec, K. (2017). Cash transfers and health: evidence from Tanzania. The World Bank Economic Review, lhx001.

Fernald, L. C., e Hidrobo, M. (2011). Effect of Ecuador's cash transfer program (Bono de Desarrollo Humano) on child development in infants and toddlers: a randomized effectiveness trial. Social Science\& Medicine, 72(9), $1437-1446$.

Fernald, L. C., Gertler, P. J., \& Neufeld, L. M. (2008). Role of cash in conditional cash transfer programmers for child health, growth, and development: an analyst is of Mexico's Oportunidades. The Lancet, 371(9615), 828-837.

Fiallos, E. E., \& Cantero, M. T. R. (2008). ¿A quién benefician los programas de salud dirigidos a los más pobres? Éxitos y fracasos. Informe SESPAS 2008. Gaceta Sanitaria, 22, 230-236

Filmer, D., \& Schady, N. (2011). Does more cash in conditional cash transfer programs always lead to larger impacts on school attendance? Journal of Development Economics, 96(1), 150-157.

Firpo, S., Pieri, R., Pedroso, E., \& Souza, A. P. (2014). Evidence of eligibility manipulation for conditional cash transfer programs. EconomiA, 15(3), 243-260.

Fiszbein, A., \& Schady, N. R. (2009). Conditional cash transfers: reducing present and future poverty. World Bank Publications.

García, S., Harker, A., \& Cuartas, J. (2019). Building dreams: The short-term impacts of a conditional cash transfer program on aspirations for higher education. International Journal of Educational Development, 64, 48-57.

Gutierrez, E., Juarez, L., \& Rubli, A. (2016). The effect of a transfer program for the elderly in Mexico City on coresiding children's school enrollment. The World Bank Economic Review, lhw012.

Jones, N., \& Samuels, F. (2015). The role of cash transfers in maximising schools' protective effects for children in extreme settings: An ecological approach. International Journal of Educational Development, 41, 217-225.

Kharroubi, E. (2007). Crises, volatility, and growth. The World Bank Economic Review, 21(3), 439-460.

Kilburn, K., Handa, S., Angeles, G., Mvula, P., \& Tsoka, M. (2017). Short-term Impacts of an Unconditional Cash Transfer Program on Child Schooling: Experimental Evidence from Malawi. Economics of Education Review. 
Kronebusch, N., \& Damon, A. (2019). The impact of conditional cash transfers on nutrition outcomes: Experimental evidence from Mexico. Economics and Human Biology, 33, 169-180.

López, R., \& Valdés, A. (2000). Fighting rural poverty in Latin America: New evidence of the effects of education, demographics, and access to land. Economic Development and Cultural Change, 49(1), 197-211.

Patanakul, P., Kwak, Y. H., Zwikael, O., \& Liu, M. (2016). What impacts the performance of large-scale government projects? International Journal of Project Management, 34(3), 452-466.

Piperata, B. A., McSweeney, K., \& Murrieta, R. S. (2016). Conditional Cash Transfers, Food Security, and Health: Biocultural Insights for Poverty-Alleviation Policy from the Brazilian Amazon, 57(6), 806-826.

Ponce, J., \& Bedi, A. S. (2010). The impact of a cash transfer program on cognitive achievement: The Bono de Desarrollo Humano of Ecuador. Economics of Education Review, 29(1), 116-125.

Ponce, P., Robles, S., Alvarado, R., \& Ortiz, C. (2019). Efecto del capital humano en la brecha de ingresos: un enfoque utilizando propensity score matching. Revista Economia y Politica, 25-47.

Rinehart, C. S. (2014). Obstacles to Uptake: The Case of Ecuador's Conditional Cash Transfer Program, the Bono de Desarrollo Humano (Doctoral dissertation, Wesleyan University).

Rineharth, C., \& Mcguire, J (2017). Obstacles to Take up: Ecuador’s Conditional Cash Transfer Program, The Bono de Desarrollo Humano. World Development, 20, 20-30

Rivera, B., Currais-Nunes, L., \& Rungo, P. (2009). Impacto de los programas de transferencia condicionada de renta sobre el estado de salud: el Programa Bolsa Familia de Brasil. Revista española de salud pública, 83(1), 85-97.

Schady, N. R., \& Araujo, M. (2006). Cash transfers, conditions, school enrollment, and child work: Evidence from a randomized experiment in Ecuador (Vol. 3). World Bank Publications.

Schady, N., \& Paxson, C. H. (2007). Does money matter? The effects of cash transfers on child health and development in rural Ecuador.

Schimit, P. H. T., Monteiro, L. H. A., \& Omar, N. (2014). Cash transfer program and education investment: A model for social evolution. Communications in Nonlinear Science and Numerical Simulation, 19(3), 570-577.

Silva, M. (2015). Conditional cash transfers and improved education quality: A political search for the policy link. International Journal of Educational Development, 45, 169-181.

Thompson, H. (2014). Cash for protection: cash transfer programs can promote child protection outcomes. Child Abuse and Neglect, Volume:38 Issue:3 Dated: March 2014 Pages:360 - 371.

Uchiyama, N. (2019). Do conditional cash transfers reduce household vulnerability? Evidence from PROGRESAOportunidades in the 2000s. EconomiA. In press.

White, J. S., \& Basu, S. (2016). Does the benefits schedule of cash assistance programs affect the purchase of temptation goods? Evidence from Peru. Journal of Heal the Economics, 46, 70-89.

(C) Universidad de Cuenca 2019

CC BY-NC-SA

\section{INFORMACIÓN ADICIONAL}

Código JEL:: C14, J01, J24, J31. 